\title{
Motor Cortex Plasticity Response to Acute Cardiorespiratory Exercise and Intermittent Theta-Burst Stimulation is Attenuated in Premanifest and Early Huntington's Disease
}

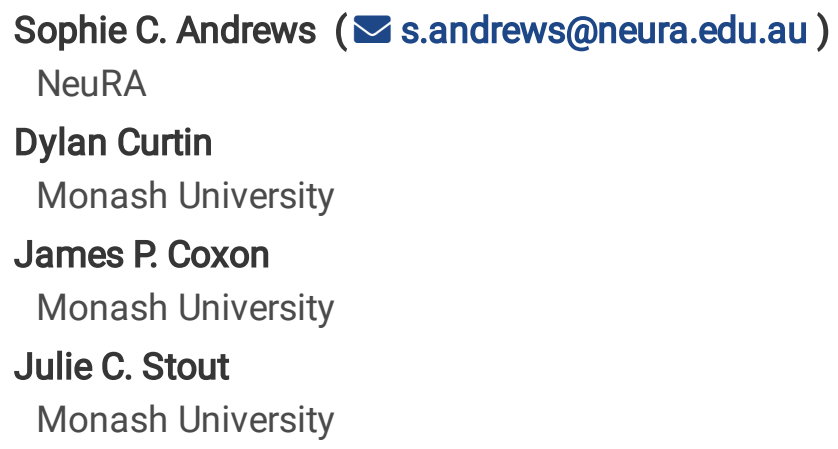

Version of Record: A version of this preprint was published at Scientific Reports on January 20th, 2022. See the published version at https://doi.org/10.1038/s41598-021-04378-2. 


\section{Abstract}

Huntington's disease (HD) mouse models suggest that cardiovascular exercise may enhance neuroplasticity and delay disease signs, however, the effects of exercise on neuroplasticity in people with HD are unknown. Using a repeatedmeasures experimental design, we compared the effects of a single bout of high-intensity exercise, moderate-intensity exercise, or rest, on motor cortex synaptic plasticity in 14 HD CAG-expanded participants (9 premanifest \& 5 early manifest) and 20 CAG-healthy control participants, using transcranial magnetic stimulation. Measures of cortico-motor excitability, short-interval intracortical inhibition and intracortical facilitation were obtained before and after a 20-minute bout of either high-intensity interval exercise, moderate-intensity continuous exercise, or rest, and again after intermittent theta burst stimulation (iTBS). HD participants showed less inhibition at baseline compared to controls. Whereas the control group showed increased excitability and facilitation following high-intensity exercise and iTBS, the HD group showed no differences in neuroplasticity responses following either exercise intensity or rest, with follow-up Bayesian analyses providing consistent evidence that these effects were absent in the HD group. These findings indicate that exercise-induced synaptic plasticity mechanisms in response to acute exercise may be attenuated in HD, and demonstrate the need for future research to further investigate exercise and plasticity mechanisms in people with HD.

\section{Introduction}

Huntington's disease (HD) is an autosomal dominant neurodegenerative disease resulting in motor dysfunction, cognitive impairment and neuropsychiatric symptoms ${ }^{1}$. Onset is typically in midlife, although the onset and course of $\mathrm{HD}$ is variable, which is proposed to be due to genetic and environmental factors, including lifestyle ${ }^{2}$. Exercise has emerged as a promising lifestyle candidate to modify disease onset and progression ${ }^{2}$. Research using HD mousemodels demonstrates that cardiovascular exercise alters biomarkers of neuroplasticity, such as brain derived neurotrophic factor (BDNF) ${ }^{3,4}$, and delays signs of disease ${ }^{5-8}$. Additionally, one retrospective study of people with HD found that a lifestyle comprising less passive activity was associated with later disease onset ${ }^{9}$. Recently, a number of exercise interventions have been trialed in HD with mixed results ${ }^{10-13}$, although one study provided preliminary evidence that a 9 month multidisciplinary intervention that included exercise increased grey matter volume and improved learning and memory ${ }^{13}$. One of the key obstacles to designing effective exercise interventions in HD is the lack of clarity regarding the mechanisms that underlie the effect of exercise in people with the HD gene.

A strong candidate mechanism is that each bout of exercise opens a window wherein the capacity for synaptic plasticity is enhanced ${ }^{14}$. Synaptic plasticity refers to the biological process that modifies the strength of communication between neurons ${ }^{15}$. In healthy adults, an acute bout of cardiovascular exercise can transiently increase synaptic plasticity in the motor cortex ${ }^{16}$. For example, 20-30 minutes of moderate-intensity continuous cycling reduces inhibition ${ }^{17,18}$, increases facilitation ${ }^{19}$, and amplifies the response to a plasticity induction protocol ${ }^{20}$. Additionally, high-intensity interval training (HIIT), where short intervals of high-intensity exercise are interspersed with periods of less intense exercise, elevates BDNF levels ${ }^{21}$, which may further benefit synaptic plasticity 212223 . Recently, we reported the first direct comparison of high-intensity interval exercise and moderate-intensity continuous exercise on synaptic plasticity in motor cortex. In a sample of 20 healthy adults, HIIT increased the response to intermittent theta burst stimulation (iTBS) compared to rest, demonstrating enhanced synaptic plasticity, with moderate-intensity exercise showing an intermediate response ${ }^{24}$. Whether high-intensity exercise is optimal to enhance synaptic plasticity in people living with neurodegenerative disease is currently unknown.

To date, no study has investigated the acute effects of exercise on motor cortex plasticity in HD. This is important because there is evidence that early disruption to cortico-striatal circuits reduces inhibition in the motor cortex 25 , and previous studies have shown that people with the CAG expansion for HD have attenuated plasticity responses to several 
non-invasive brain stimulation protocols ${ }^{26-29}$. Thus, exercise presents a possible intervention for people with the HD CAG expansion to boost neuroplasticity, optimize cognitive reserve, and delay symptom onset or slow progression ${ }^{30}$. The aim of the current study was therefore to investigate whether a single bout of either HIIT or moderate-intensity continuous exercise enhances the response to iTBS in premanifest and early manifest HD, in comparison to an HD-CAG healthy control group. Using our established transcranial magnetic stimulation (TMS) methodology ${ }^{24}$, we measured how exercise intensity alters inhibition and excitation in the motor cortex, and synaptic plasticity as induced by iTBS. We hypothesized that similar to healthy controls, HD gene-expanded participants would show the largest enhancement of synaptic plasticity following high-intensity, rather than moderate-intensity exercise (compared to rest). We also tested the hypothesis that baseline alterations to motor cortex neurophysiology (e.g., reduced inhibition and increased facilitation) would attenuate this effect.

\section{Methods}

\section{Participants}

The sample comprised 20 CAG healthy control participants (Control Group), and 14 HD CAG gene-expanded participants (HD Group; 9 premanifest, 5 early manifest) recruited from the ENRU-Stout HD database held at Monash University, and the Statewide Progressive Neurological Disease Service at Calvary Health Care Bethlehem in Melbourne, Australia. Participants were aged between 21 and 70 years, right-handed, and were screened for contraindications to TMS ${ }^{31}$ and exercise (e.g., cardiovascular disease, uncontrolled hypertension) ${ }^{32}$. Exclusion criteria were a history of any psychiatric or neurological illness (except HD for the HD group), seizure, any serious medical conditions, or current pregnancy.

The HD Group included people with genetically-confirmed expansion of the HD CAG repeat sequence ( $\geq 39$ CAG repeats). Participants were classified as premanifest if they had never received a clinical diagnosis of HD, and as early HD if they had received a clinical diagnosis of HD. Disease Burden Score (calculated as age x [CAG-35.5]) ranged from 91-441 33 , equating to estimated years to onset ranging from 0 to 43 years, and Total Functional Capacity (TFC) on the Unified Huntington's Disease Rating Scale ${ }^{34}$ (UHDRS) ranged from 11-13 ${ }^{35}$, indicating no more than mild functional impairment. For those who also participated in the longitudinal, observational Enroll-HD study $(n=13)$, UHDRS Total Motor Score was obtained from their most recent annual visit. Handedness was self-reported using the Edinburgh Handedness Inventory ${ }^{36}$, and current physical activity levels were assessed using the International Physical Activity Questionnaire (IPAQ) ${ }^{37}$. Groups did not differ in age, gender distribution, years of education, self-reported anxiety or depression, or current levels of physical activity (see Table 1). The study was approved by the Human Research Ethics Committees of Monash University and Calvary Health Care Bethlehem in Melbourne, Australia, and all participants gave written informed consent. All procedures were performed in accordance with the World Medical Association Declaration of Helsinki. 
Table 1

Participant characteristics

\begin{tabular}{|c|c|c|c|c|}
\hline & HD Gene-Positive & Healthy Controls & Test Statistic & $p$-value \\
\hline $\mathrm{N}$ & 14 & 20 & & \\
\hline Age (years) & 39.71 (13.73), 26-70 & 35.15 (13.25), $21-64$ & $T_{32}=-.97$ & .34 \\
\hline Women & $10(71 \%)$ & $12(60 \%)$ & $\chi_{1}^{2}=.47$ & .49 \\
\hline Years of Education & $15.86(2.31)$ & $16.85(3.05)$ & $T_{32}=1.03$ & .31 \\
\hline CAG repeat length & 41.71 (1.82), 39-46 & - & & \\
\hline Disease- burden score & 246 (104), 91-441 & - & & \\
\hline UHDRS-TMS $(n=13)$ & 4.92 (8.98), 0-25 & - & & \\
\hline UHDRS-TFC & $12.46(0.87), 11-13$ & - & & \\
\hline HADS Anxiety & 5.57 (3.39), 0-11 & $4.90(3.34), 0-13$ & $T_{32}=-.57$ & .57 \\
\hline HADS Depression & $3.42(3.18), 0-10$ & $2.65(2.30), 0-8$ & $T_{32}=-.83$ & .41 \\
\hline Handedness, EHI & $+79.62(15.13)$ & $+80.20(13.27)$ & $T_{31}=.15$ & .88 \\
\hline BMI & $24.52(2.41)$ & $23.73(4.10)$ & $T_{32}=-.64$ & .52 \\
\hline IPAQ & $6949(4916)$ & $4681(2287)$ & $T_{15.42}=-1.56$ & .14 \\
\hline Resting Heart Rate, BPM & $63(7.38)$ & $68(12.51)$ & $T_{31.28}=1.43$ & .16 \\
\hline Rest Threshold (\%MSO) & $61(10.96)$ & $66(10.26)$ & $F_{1,32}=1.65$ & .21 \\
\hline Active Threshold (\%MSO) & $49(8.23)$ & $52(9.28)$ & $F_{1,32}=1.28$ & .26 \\
\hline Test Stimulus (\%MSO) & $78(15.55)$ & $79(12.40)$ & $F_{1,32}=.03$ & .86 \\
\hline Conditioning Stimulus (\%MSO) & $41(7.65)$ & $41(8.18)$ & $F_{1,32}=.02$ & .90 \\
\hline iTBS Stimulus (\%MSO) & $29(5.09)$ & $31(4.97)$ & $F_{1,32}=.69$ & .41 \\
\hline Baseline 1mV (NC) & $.95(.25)$ & $1.07(.28)$ & $F_{1,32}=1.70$ & .20 \\
\hline Baseline SICI (C/NC Ratio) & $.61(.24)$ & $.50(.07)$ & $F_{1,32}=5.11$ & $.03 *$ \\
\hline Baseline ICF (C/NC Ratio) & $1.19(.33)$ & $1.18(.40)$ & $F_{1,32}=.005$ & .94 \\
\hline Baseline E-I Ratio & $2.79(2.29)$ & $2.25(1.11)$ & $F_{1,32}=.84$ & .37 \\
\hline
\end{tabular}

Data are mean (SD), range or number (\%). UHDRS-TMS - Total Motor Score: Possible scores range from 0-124; UHDRS-TFC - Total Functional Capacity: Possible scores range from 0-13; UHDRS-TMS - Total Motor Score: Possible scores range from 0-124; HADS - Hospital Anxiety and Depression Scale; EHI - Edinburgh Handedness Inventory, [range: -100 (left-handed) to + 100 (right-handed)]; BMI - Body Mass Index; IPAQ - International Physical Activity Questionnaire (higher scores indicate higher levels of physical activity); BPM - beats per minute; RMT - resting motor threshold; MSO - Maximum Stimulator Output, AMT - active motor threshold with monophasic stimulation; TS - test 
stimulus; CS - conditioning stimulus; iTBS = intermittent theta burst stimulation; NC - Non-conditioned; C - Conditioned; $\mathrm{SICl}$ - Short Interval Intracortical Inhibition; ICF - Intracortical Facilitation; E-I - Excitation-Inhibition ratio.

\section{Study Design and Methods}

Each participant (HD, controls) completed three sessions separated by at least 72 hours, with session order counterbalanced within each group. Participants were instructed to refrain from moderate and vigorous physical activity for 24 hours prior to each session.

Session protocol. Participants were fitted with a chest-strap heart-rate monitor (Polar H7, Polar Electro), then seated for the Baseline TMS assessment. Next they completed 20 minutes of either high-intensity interval stationary cycling, moderate-intensity continuous stationary cycling, or rest, followed by a 10-min cool down, the post-exercise TMS assessment, iTBS, then Post-iTBS TMS assessments at 5, 15 and 25 minutes (see Fig. 1).

\section{TMS}

We recorded several common single- and paired-pulse TMS measures, including corticomotor excitability (CME), shortinterval intracortical inhibition (SICI), and intracortical facilitation (ICF) ${ }^{24,38}$. SICl is a measure of gamma-amino butyric acid (GABA) receptor $A$ inhibition, whereas ICF has been linked to both the excitatory neurotransmitter glutamate and catecholamine function ${ }^{39}$. Obtaining both $\mathrm{SICI}$ and ICF enables assessment of the excitation-inhibition balance 24,40 .

The TMS protocol used in this study has been described elsewhere ${ }^{24}$. Briefly, participants were seated with both hands resting on a pillow on their laps. Surface electromyography (EMG) was recorded from first dorsal interosseous (FDI) of the right hand in a belly-tendon montage. Monophasic TMS pulses were applied to left primary motor cortex using a $70 \mathrm{~mm}$ diameter figure-of-eight coil connected to a MagVenture MagPro X100 stimulator (MagVenture Ltd.). At the beginning of each session we determined resting motor threshold, active motor threshold, and the test stimulus intensity required to produce a stable MEP of $\sim 1 \mathrm{mV}$. Paired-pulse TMS was used to assess SICI and ICF, with the sub-threshold conditioning stimulus preceding the supra-threshold $\sim 1 \mathrm{mV}$ test stimulus by $2 \mathrm{~ms}$ and $12 \mathrm{~ms}$, respectively. The conditioning stimulus intensity was titrated to produce $50 \%$ inhibition of the non-conditioned (NC) MEP then held constant for all SICl and ICF measures. To ensure valid comparison of paired-pulse measures across different timepoints, where necessary we adjusted the test stimulus, so that test MEP sizes were matched to within $30 \%$ of the comparison time-point (i.e. Post-Exercise was matched to Baseline, Post-iTBS was matched to Post-Exercise) ${ }^{22,24}$. For all single- and paired-pulse measurements, 16 stimuli were delivered at 5 second intervals.

iTBS was applied to the same motor cortex site, using the same TMS coil and stimulator, following standard procedure $^{41}$. The iTBS intensity was set at $80 \%$ of biphasic active threshold, and three high frequency (50Hz) biphasic pulses were delivered every 200 ms for 2 s, repeated every 10 s for 20 repetitions.

\section{Exercise}

Participants completed the exercise sessions on a stationary cycle ergometer (Wattbike). We asked participants to refrain from gripping the handlebar of the bike during exercise, and heart rate was continuously monitored. The intensity of each exercise session was tailored to individual participants based on their estimated heart rate reserve (HRR), which is an indication of the functional range of the heart during exercise. Resting heart rate was measured while participants were seated and HRR was computed by subtracting resting heart rate from age-predicted maximum heart rate (220 current age). 
The high-intensity exercise protocol comprised alternating 3 min bouts of cycling at $50 \% \mathrm{HRR}$, with 2 min bouts of cycling at up to $90 \%$ HRR, for a total duration of 20 minutes ${ }^{24}$. The moderate-intensity exercise protocol comprised 20 mins cycling at 50\% HRR. For the rest session, participants sat quietly for 20 minutes. Participants self-reported their perceived exertion (RPE) using Borg's scale (ranging from 6, no exertion at all, to 20, maximal exertion) ${ }^{42}$. Participants completed a two-minute warm-up and two-minute cool-down and the beginning and end of each exercise session, followed by a 10-minute quiet rest period before the TMS protocol resumed.

Table 2 shows the effectiveness of our exercise intensity manipulation in each group. During the final epoch of the highintensity exercise session, the HD and control groups reached a maximum of $93 \%$ and $91 \% \mathrm{HRR}$, respectively. In contrast, for the moderate intensity protocol, on average the HD and control groups exercised at $52 \%$ and $50 \%$ of $\mathrm{HRR}$. During the high-intensity session, there were no differences between the groups on power, power:weight ratio, cadence, HRR, or RPE for any of the epochs (all $p s>.08$ ). During the moderate-intensity session, there were no differences between groups for power, power:weight ratio, cadence or HRR (all ps>.14;); however, the control group reported a higher perceived exertion than the HD group on average during the moderate intensity session $t_{28.7}=2.99, p=.006$ ).

Table 2

Effects of High-intensity Interval Training and Moderate-intensity Continuous Training on Exercise Performance

\begin{tabular}{|c|c|c|c|c|c|c|c|c|c|c|}
\hline \multirow{2}{*}{$\begin{array}{l}\text { Session } \\
\text { HIIT }\end{array}$} & \multicolumn{2}{|c|}{ Power (Watts) } & \multicolumn{2}{|c|}{$\begin{array}{l}\text { Power:Weight } \\
\text { (Watts:kg) }\end{array}$} & \multicolumn{2}{|c|}{ Cadence (RPM) } & \multicolumn{2}{|l|}{ \%HRR } & \multicolumn{2}{|l|}{ RPE } \\
\hline & Controls & HD & Controls & HD & Controls & HD & Controls & HD & Controls & HD \\
\hline $\begin{array}{l}0-3 \\
\min \end{array}$ & $\begin{array}{l}68 \\
(17.20)\end{array}$ & $\begin{array}{l}68 \\
(24.45)\end{array}$ & $\begin{array}{l}1.00 \\
(0.24)\end{array}$ & $\begin{array}{l}1.05 \\
(0.31)\end{array}$ & $\begin{array}{l}76 \\
(7.93)\end{array}$ & $\begin{array}{l}73 \\
(11.92)\end{array}$ & $\begin{array}{l}43 \% \\
(0.05)\end{array}$ & $\begin{array}{l}45 \% \\
(0.07)\end{array}$ & $\begin{array}{l}10 \\
(1.47)\end{array}$ & $\begin{array}{l}10 \\
(1.73)\end{array}$ \\
\hline $\begin{array}{l}4-5 \\
\min \end{array}$ & $\begin{array}{l}106 \\
(34.69)\end{array}$ & $\begin{array}{l}105 \\
(31.64)\end{array}$ & $\begin{array}{l}1.56 \\
(0.45)\end{array}$ & $\begin{array}{l}1.61 \\
(0.33)\end{array}$ & $\begin{array}{l}90 \\
(8.96)\end{array}$ & $\begin{array}{l}88 \\
(13.34)\end{array}$ & $\begin{array}{l}62 \% \\
(0.07)\end{array}$ & $\begin{array}{l}64 \% \\
(0.12)\end{array}$ & $\begin{array}{l}13 \\
(1.43)\end{array}$ & $\begin{array}{l}13 \\
(2.05)\end{array}$ \\
\hline $\begin{array}{l}6-8 \\
\min \end{array}$ & $\begin{array}{l}71 \\
(17.88)\end{array}$ & $\begin{array}{l}75 \\
(30.04)\end{array}$ & $\begin{array}{l}1.05 \\
(0.26)\end{array}$ & $\begin{array}{l}1.16 \\
(0.39)\end{array}$ & $\begin{array}{l}77 \\
(8.05)\end{array}$ & $\begin{array}{l}75 \\
(12.62)\end{array}$ & $\begin{array}{l}52 \% \\
(0.05)\end{array}$ & $\begin{array}{l}52 \% \\
(0.06)\end{array}$ & $\begin{array}{l}11 \\
(1.30)\end{array}$ & $\begin{array}{l}11 \\
(1.36)\end{array}$ \\
\hline $\begin{array}{l}9-10 \\
\min \end{array}$ & $\begin{array}{l}111 \\
(32.37)\end{array}$ & $\begin{array}{l}114 \\
(36.82)\end{array}$ & $\begin{array}{l}1.63 \\
(0.44)\end{array}$ & $\begin{array}{l}1.73 \\
(0.41)\end{array}$ & $\begin{array}{l}95 \\
(7.88)\end{array}$ & $\begin{array}{l}90 \\
(15.47)\end{array}$ & $\begin{array}{l}73 \% \\
(0.04)\end{array}$ & $\begin{array}{l}74 \% \\
(0.11)\end{array}$ & $\begin{array}{l}13 \\
(1.66)\end{array}$ & $\begin{array}{l}13 \\
(2.01)\end{array}$ \\
\hline $\begin{array}{l}11-13 \\
\min \end{array}$ & $\begin{array}{l}73 \\
(18.62)\end{array}$ & $\begin{array}{l}76 \\
(30.07)\end{array}$ & $\begin{array}{l}1.07 \\
(0.25)\end{array}$ & $\begin{array}{l}1.17 \\
(0.37)\end{array}$ & $\begin{array}{l}78 \\
(8.26)\end{array}$ & $\begin{array}{l}75 \\
(12.78)\end{array}$ & $\begin{array}{l}57 \% \\
(0.04)\end{array}$ & $\begin{array}{l}58 \% \\
(0.07)\end{array}$ & $\begin{array}{l}11 \\
(1.34)\end{array}$ & $\begin{array}{l}11 \\
(1.05)\end{array}$ \\
\hline $\begin{array}{l}14-15 \\
\min \end{array}$ & $\begin{array}{l}122 \\
(32.18)\end{array}$ & $\begin{array}{l}126 \\
(47.61)\end{array}$ & $\begin{array}{l}1.80 \\
(0.47)\end{array}$ & $\begin{array}{l}1.90 \\
(0.54)\end{array}$ & $\begin{array}{l}101 \\
(17.87)\end{array}$ & $\begin{array}{l}96 \\
(14.92)\end{array}$ & $\begin{array}{l}82 \% \\
(0.04)\end{array}$ & $\begin{array}{l}80 \% \\
(0.10)\end{array}$ & $\begin{array}{l}14 \\
(1.63)\end{array}$ & $\begin{array}{l}15 \\
(1.51)\end{array}$ \\
\hline $\begin{array}{l}16-18 \\
\min \end{array}$ & $\begin{array}{l}72 \\
(17.83)\end{array}$ & $\begin{array}{l}73 \\
(29.05)\end{array}$ & $\begin{array}{l}1.06 \\
(0.24)\end{array}$ & $\begin{array}{l}1.11 \\
(0.37)\end{array}$ & $\begin{array}{l}77 \\
(9.86)\end{array}$ & $\begin{array}{l}75 \\
(13.50)\end{array}$ & $\begin{array}{l}62 \% \\
(0.06)\end{array}$ & $\begin{array}{l}59 \% \\
(0.07)\end{array}$ & $\begin{array}{l}12 \\
(1.60)\end{array}$ & $\begin{array}{l}12 \\
(1.48)\end{array}$ \\
\hline $\begin{array}{l}19-20 \\
\min \end{array}$ & $\begin{array}{l}156 \\
(61.45)\end{array}$ & $\begin{array}{l}165 \\
(59.30)\end{array}$ & $\begin{array}{l}2.30 \\
(0.86)\end{array}$ & $\begin{array}{l}2.51 \\
(0.63)\end{array}$ & $\begin{array}{l}105 \\
(12.41)\end{array}$ & $\begin{array}{l}108 \\
(15.87)\end{array}$ & $\begin{array}{l}91 \% \\
(0.06)\end{array}$ & $\begin{array}{l}93 \% \\
(0.15)\end{array}$ & $\begin{array}{l}18 \\
(1.32)\end{array}$ & $\begin{array}{l}17 \\
(1.63)\end{array}$ \\
\hline MICT & Controls & HD & Controls & HD & Controls & HD & Controls & HD & Controls & HD \\
\hline $\begin{array}{l}0-20 \\
\min \end{array}$ & $\begin{array}{l}75 \\
(19.14)\end{array}$ & $\begin{array}{l}76 \\
(27.39)\end{array}$ & $\begin{array}{l}1.09 \\
(0.23)\end{array}$ & $\begin{array}{l}1.14 \\
(0.37)\end{array}$ & $\begin{array}{l}79 \\
(9.78)\end{array}$ & $\begin{array}{l}77 \\
(10.87)\end{array}$ & $\begin{array}{l}50 \% \\
(0.05)\end{array}$ & $\begin{array}{l}52 \% \\
(0.03)\end{array}$ & $\begin{array}{l}12 \\
(1.18)\end{array}$ & $\begin{array}{l}11 \\
(0.54)\end{array}$ \\
\hline
\end{tabular}

Data are mean (SD). HIIT: High-intensity interval training; MICT: Moderate-intensity continuous training; RPM: Revolutions per minute; \%HRR: Percentage of Heart Rate Reserve; and RPE: Rating of Perceived Exertion.

\section{Data Analysis}


EMG recordings were inspected offline, and trials discarded if contaminated with muscle activity in the 100ms before the TMS pulse, or if root mean square EMG was $\geq 10 \mu \mathrm{V}$, because muscle activity at the time of the TMS pulse is known to influence MEP amplitudes. For CME, MEP amplitudes were normalized to the mean MEP at the post-exercise timepoint (i.e., a value of 1 was assigned to the post-exercise time-point and all other values were expressed relative to this value $^{41}$ ). For paired-pulse measures, the conditioned (C) MEP was expressed as a ratio of the non-conditioned (NC) MEP $(\mathrm{C} / \mathrm{NC})$. To reduce the influence of outliers, we used an a priori trimmed mean procedure, in which the highest and lowest values of each set of 16 MEPs were discarded ${ }^{43}$. Any univariate outliers identified $(z \pm 3.29$; four in the HD group and four in the Control group) were adjusted to a unit higher than the next most extreme score in their respective condition. Due to technical constraints, two values were not obtained at Baseline for a control participant in the Rest condition, and two values were not obtained at the Post-iTBS 15-minute time-point for an HD participant in the Rest condition. These values were imputed using mean value replacement. Prior to analysis, a visual inspection of histograms showed positive skew for SICI and ICF variables. To satisfy the normality assumption for statistical analyses, we transformed these variables using a natural logarithm, however, untransformed data are presented in Figures.

Frequentist analyses were conducted using the Statistical Package for the Social Sciences (Version 25; Chicago, IL, USA), with alpha set to 0.05 . To determine differences between groups in the absence of exercise, we compared the HD and control groups on TMS measures at baseline, by undertaking two-way mixed model analysis of variance (ANOVA), with Exercise Session as the within-subjects variable and Group as the between-subjects variable. Separate ANOVAs were conducted for each of the TMS measures of interest (Rest threshold, Active threshold, CME, SICl, ICF). Where appropriate, Greenhouse-Geisser epsilon was used to correct for violations of sphericity. To examine differences in synaptic plasticity following exercise across groups, we used three-way mixed model ANOVAs, where Exercise Session and Time were within-subjects factors, and Group was the between subjects factor. Separate ANOVAs were conducted for the TMS measures of CME, SICI and ICF. Significant main effects and interactions were examined further using oneway ANOVAs and pairwise comparisons. To assess magnitude of effects, we calculated partial eta-squared $\left(\eta_{p}{ }^{2}\right)$.

Given our small sample size, and to more directly compare the effect of exercise condition on change in neuroplasticity measures (CME, SICI, ICF) post iTBS within each group, Bayesian analysis is also reported. Bayesian analyses are helpful in the context of non-significant results, as they provide quantification of evidence in favor of the null hypothesis, and therefore indicate whether the observed data truly provide evidence of absence, or simply indicate an absence of evidence ${ }^{44,45}$. In order to assess the strength of the evidence for the presence or absence of an effect of exercise on the neuroplasticity measures (CME, SICI and ICF) within each group separately, one-way repeated measures Bayesian ANOVAs were run for each group, where Exercise Session was the within-subjects factor, using JASP software ${ }^{46}$. Specifically, we calculated Bayes Factors corresponding to evidence in favor of $H_{1}$ relative to the $H_{0}\left(B F_{10}\right)$, using default priors for ANOVA ${ }^{47}$. By convention, only Bayes factor values below 0.33 or above 3 are considered noteworthy, with a $\mathrm{BF}_{10}$ value $\geq 3$ indicating that the alternative hypothesis is $\geq 3$ times more likely than the null hypothesis, and conversely a $\mathrm{BF}_{10}$ value $\leq 0.33$ indicating the null hypothesis is $\leq 3$ times more likely than the alternative hypothesis, i.e., at least moderate evidence in favor of the alternative, or null hypothesis, respectively ${ }^{48,49}$. Values between 0.33 and 3 are considered inconclusive and only anecdotal evidence in favor of either hypothesis. ANOVA results with a Bayes Factors $\geq 3$ were followed up with post hoc comparisons based on the default Bayesian t-test with a Cauchy prior.

\section{Results}

Baseline motor cortex neurophysiology. The HD and control groups were matched in terms of baseline resting and active motor cortex thresholds, and facilitation (ICF), with no significant main effects of Group or Session, and no interaction effects (Table 1 displays main effects of Group results for these variables). HD participants showed 
significantly less inhibition (SICl) at baseline than controls across all sessions, represented by a significant main effect of $\operatorname{Group}\left(\mathrm{F}_{1,32}=5.11, p=.03, \eta_{\mathrm{p}}^{2}=.14\right)$.

Cortico-motor excitability. We examined the effect of exercise on CME by normalizing the average post-iTBS MEP to the post-exercise time-point. Two-way ANOVA revealed a significant Group x Session interaction, $\left(F_{2,64}=3.72, p=.03, \eta_{p}{ }^{2}\right.$ $=.10$ ), where the Control group showed significantly larger MEPs following iTBS in the high-intensity exercise condition than the HD group $(t(32)=2.39, p=.02)$, but there were no significant differences in MEPs between groups in either the Moderate intensity $(t(32)=.41, p=.70)$ or Rest condition $(t(32)=-.83, p=.42)$ (Fig. 2a). For the HD group, the Bayesian one-way repeated measures ANOVA provided moderate evidence in favor of the null hypothesis, that is, that neither highnor moderate-intensity exercise boosted CME post-iTBS $\left(B_{10}=.18\right.$; null hypothesis approximately 5.4 times more likely than the alterative hypothesis). In contrast, the control group showed very strong evidence of a significant difference between sessions, $\left(\mathrm{BF}_{10}=36.23\right)$, with post-hoc comparisons revealing strong evidence that high intensity exercise increased $\mathrm{CME}$ post-iTBS compared to rest $\left(\mathrm{BF}_{10}=20.15\right)$. In contrast, there was no convincing evidence that highintensity exercise enhanced $C M E$ compared to moderate-intensity exercise $\left(\mathrm{BF}_{10}=1.35\right)$, nor that moderate-intensity exercise increased $\mathrm{CME}$ in comparison to rest $\left(\mathrm{BF}_{10}=.68\right)$.

Short-interval Intracortical Inhibition. A 3 Session x 5 Time x 2 Group mixed model ANOVA revealed a significant reduction in inhibition following high- and moderate-intensity exercise and iTBS in the control group but not the HD group, reflected by a significant Session $x$ Group interaction effect $\left(F_{2,64}=3.45, p=.04, \eta_{p}{ }^{2}=.10\right)$, and a significant main effect of Time $\left(F_{4,128}=3.35, p=.01, \eta_{p}^{2}=.10\right)$. There were no main effects of Session, or Group, or interaction effects between Session x Time, Time x Group, or Session x Time x Group. The change in SICl from the Pre-Exercise time-point to the average of the post-iTBS time-points for each group is shown in Fig. 2b. There were no significant differences between groups for any of the conditions (Rest: $(t(32)=1.05, p=.30)$; Moderate-intensity condition: $(t(32)=1.1, p=.27)$; High-intensity condition: $(t(32)=1.5, p=.14)$ ). For the HD group, Bayesian one-way repeated-measures ANOVA provided moderate evidence in favor of the null hypothesis, that is, that neither high-nor moderate-intensity exercise reduced SICI post iTBS $\left(\mathrm{BF}_{10}=.27\right)$. For the control group, the evidence that the change in SICl differed between sessions was inconclusive $\left(\mathrm{BF}_{10}=1.20\right)$.

Intracortical Facilitation. A 3 Session x 5 Time x 2 Group mixed model ANOVA revealed a significant increase in facilitation following high-intensity exercise and iTBS in the control group but not the HD group, reflected by a significant Session $x$ Group interaction effect $\left(F_{2,64}=3.93, p=.03, \eta_{p}{ }^{2}=.11\right)$, and a significant main effect of Time $\left(F_{4,128}=5.83, p<.001, \eta_{p}{ }^{2}=.15\right)$. There were no main effects of Session, or Group, or interaction effects between Session $x$ Time, Time $x$ Group, or Session $x$ Time $x$ Group. The change in ICF from the Pre-Exercise time-point to the average of the post-iTBS time-points for each group is shown in Fig. 2c. There was significantly more facilitation following high-intensity exercise and iTBS in the control group in comparison to the HD group, $(t(32)=2.13, p=.04)$, but there were no between group differences in the moderate exercise $(t(32)=-.89, p=.38)$ or rest $(t(32)=.09, p=.93)$ conditions. For the HD group, Bayesian one-way ANOVA again revealed moderate evidence in favor of the null hypothesis, indicating neither the high- nor moderate-intensity exercise had any effect on ICF post iTBS $\left(B_{10}=.27\right)$. In contrast, for the control group, there was strong evidence in favor of the alternative hypothesis, that ICF differed across groups $\left(\mathrm{BF}_{10}=14.39\right)$, with moderate evidence that high-intensity exercise induced a larger increase in ICF following iTBS than rest $\left(\mathrm{BF}_{10}=6.20\right)$, but no conclusive evidence that high-intensity exercise induced are larger increase in ICF following iTBS than moderate-intensity exercise (BF10 = 2.38). In comparison, there was moderate evidence that moderate-intensity exercise had no effect on ICF, in comparison to rest $\left(\mathrm{BF}_{10}=.24\right)$. 
Relationships between demographic and clinical characteristics and TMS outcomes. We investigated relationships between age, self-reported physical activity levels, mood symptoms, and delta change values (Post-iTBS - Baseline) in the TMS measures (CME, SICl, or ICF), using Pearson correlations (Spearman correlations for non-normally distributed variables) and a more conservative significance level of $p<.01$ due to multiple comparisons. There were no significant relationships between these variables in either group across any of the three conditions. There were also no significant relationships between DBS and any of the TMS measures in the HD group.

\section{Discussion}

Contrary to our first hypothesis, the premanifest and early HD participants did not show enhancement of synaptic plasticity following high-intensity interval exercise, in comparison to either moderate intensity exercise or rest. Specifically, the HD group showed no increases in cortico-motor excitability, glutamatergic facilitation, or decreases in $\mathrm{GABA}_{A}$ ergic inhibition following either high- or moderate-intensity exercise, a finding which was further supported by the follow-up Bayesian analyses. The HD group showed lower inhibition at baseline which may have attenuated the effect of exercise on plasticity, consistent with our second hypothesis. Taken together, the current findings indicate that HD is associated with an abnormal, attenuated plasticity response to an acute bout of cardiorespiratory exercise in premanifest and early HD, which has implications for the design of exercise interventions in this population.

Several possible mechanisms may account for the absence of exercise induced plasticity in the HD group. The first is that the HD group showed low baseline levels of $\mathrm{GABA}_{\mathrm{A}}$ ergic intracortical inhibition compared to controls, which has been observed in several previous studies ${ }^{27,28,50}$. In healthy adults, exercise transiently reduces $\mathrm{GABA}_{A}$ ergic intracortical inhibition $^{17,18}$. In HD, due to homeostatic mechanisms, the already reduced SICI in HD at baseline may have precluded further reductions in response to exercise ${ }^{51}$. However, baseline levels of corticomotor excitability and intracortical facilitation were not different than controls. Given the HD group also did not show the expected plasticity response for these variables, homeostatic mechanisms are unlikely to wholly account for the lack of responsivity of neuroplasticity to exercise.

The absence of the normal neuroplasticity response to exercise may be attributable to altered or dysregulated dopamine signaling, as well as reduced production of BDNF in HD. Specifically, the indirect (D2) dopaminergic pathway within the striatum is affected early in $\mathrm{HD}$, which connects to the motor cortex via the nigrostriatal pathway ${ }^{52}$, and the dopamine D2 receptor has been shown to mediate the effects of exercise on motor learning ${ }^{53}$. Mutant huntingtin has also been found to decrease the level of BDNF and its receptor tropomyosin-related kinase B (TrkB) in human and mouse brains, and reduced release of BDNF has been observed in cortical neurons of an HD mouse-model ${ }^{54}$. This is important, because in healthy adults, acute exercise triggers the release of lactate, dopamine, and the synthesis and release of $\mathrm{BDNF}^{55}$, which are associated with decreased cortical inhibition and corresponding increase in neuroplasticity ${ }^{55,56}$. The disruption of these processes in early HD, therefore, may interfere with the usual increases in dopamine and BDNF observed following acute exercise, and result in reduced motor cortex plasticity.

These findings have important implications for studies of exercise in HD, as attenuated brain responses to exercise may contribute to the mixed outcomes reported for exercise interventions in HD to date. Although some non-randomized controlled trials of exercise interventions have reported promising results, a recent meta-analysis of motor and cognitive effects from randomized controlled trials indicated no significant effects from the interventions on either the primary outcome (UHDRS motor score) or secondary outcomes (cognitive, health status or physical) ${ }^{11}$. The current findings indicate that more research is urgently needed to understanding under what circumstances exercise may elicit an optimal neurophysiological response in HD (e.g., by investigating response to different types of exercise, or if there are 
effects over the longer-term following multiple exercise sessions), to inform the future direction of exercise intervention research in this population.

Our findings contrast with reports of exercise effects on the brain in HD mouse models. For example, wheel running in R6/1 transgenic HD mice increases BDNF gene expression ${ }^{3,4}$ and delays onset of motor signs ${ }^{7}$, whereas treadmill exercise in CAG140 knock-in HD mice restores dopamine D2 receptor expression ${ }^{6}$. The exercise interventions in these studies were long-term, however, rather than a single bout of exercise as implemented in the current study. In HD, longerterm exercise may be needed to have potent beneficial effects on brain chemistry, such as BDNF and dopamine levels.

Our study included people with the HD CAG expansion in very early stage, as much as 43 years before predicted onset, but also participants who had already been diagnosed. Manifest HD participants may have had too much neurodegeneration to respond optimally to exercise. However, we did not find support for this possibility, in that disease burden score in our HD sample was not associated with the size of plasticity response following exercise. Thus neuroplasticity in HD does not necessarily track with disease progression.

Unlike the control group, who showed an increased facilitatory response to iTBS following high-intensity exercise, HD participants did not show any change to iTBS response following either exercise intensity. Only one previous study has utilized a theta-burst paradigm within a HD population. Orth et al. investigated responses to continuous TBS (cTBS) in a mixed premanifest and manifest HD group, and found that cTBS had no effect on inhibition in HD, whereas cTBS resulted in significantly increased inhibition in the control group ${ }^{26}$. Our current study extends this to show that in premanifest and early HD, there was no detectable effect of iTBS on excitability, inhibition or facilitation, either alone or primed with moderate- or high-intensity exercise. This suggests that these neuroplasticity mechanisms are affected early on in the disease course of HD, which has important implications for the consideration of non-invasive brain stimulation interventions in HD.

The current study has a number of limitations. We did not include an objective measure of cardiorespiratory fitness (e.g., $\mathrm{VO}_{2 \text { max }}$ ), and therefore we could not examine any relationships between physical fitness and synaptic plasticity. However, groups did not differ on self-reported physical activity levels, resting heart rate, or objective measures of exercise performance, suggesting our groups were reasonably well matched. We also did not assess the potential role of BDNF genotype as a mediator of the plasticity response within the HD group, due to sample size limitations. Our group and others have previously found BDNF genotype to mediate the plasticity response to iTBS in healthy adults ${ }^{24,57}$. Further, our small sample size, particularly in the HD group, means that we may have been underpowered to detect an effect of exercise on neuroplasticity in the HD sample. Our follow up Bayesian analyses, however, provide moderate evidence that the results likely reflect a true absence of effect of exercise.

\section{Conclusions}

In conclusion, the current study is the first to investigate the acute effects of exercise and iTBS on measures of motor cortex plasticity in premanifest and early HD. In contrast to the control group, we found no effect of either high- or moderate-intensity exercise and iTBS on the individual plasticity measures in the HD participants. These findings call into question the assumption of the benefits of exercise to the brain in HD, and demonstrate the need for future research to better understand exercise and plasticity mechanisms in people with the HD gene expansion, as well as investigate the effect of alternative exercise and non-invasive brain stimulation protocols on neuroplasticity in HD.

\section{Declarations}

\section{Acknowledgements}

Page $10 / 15$ 
This study was funded by a Huntington's Disease Society of America Human Biology Project Grant. Sophie Andrews received salary support from the Huntington's Disease Society of America. We thank the participants for their time and effort which made this study possible. We also thank Jaeger Wongtrakun, Joshua Hendrikse, Melissa Pelly and Claire Cadwallader for their assistance with data collection on this project.

\section{Author Contributions Statement}

SCA, JPC AND JCS conceptualised and designed the study. SCA and DC acquired the data. SCA ran the analysis, interpreted the data, and drafted the manuscript. All authors reviewed and edited the manuscript.

\section{Declaration of Conflicting Interests}

Prof. Stout has served on Scientific Advisory Boards (Teva-Australia, Spark Therapeutics), and has performed consulting services for the CHDI Foundation, uniQure NV, and Triplet Therapeutics. She is a director with financial responsibilities for Zindametrix Pty Ltd, which has held contracts in the past twelve months with Vaccinex, uniQure NV, Triplet Therapeutics, and Voyager Therapeutics. Dr. Andrews, Mr. Curtin and Dr. Coxon declare no potential conflict of interest.

\section{References}

1. Walker, F.O. Huntington's disease. Lancet 369, 218-228 (2007).

2. Mo, C., Hannan, A.J. \& Renoir, T. Environmental factors as modulators of neurodegeneration: insights from geneenvironment interactions in Huntington's disease. Neurosci Biobehav Rev 52, 178-192 (2015).

3. Zajac, M.S., et al. Wheel running and environmental enrichment differentially modify exon-specific BDNF expression in the hippocampus of wild-type and pre-motor symptomatic male and female Huntington's disease mice. Hippocampus 20, 621-636 (2010).

4. Pang, T.Y.C., Stam, N.C., Nithianantharajah, J., Howard, M.L. \& Hannan, A.J. Differential effects of voluntary physical exercise on behavioral and brain-derived neurotrophic factor expression deficits in Huntington's disease transgenic mice. Neuroscience 141, 569-584 (2006).

5. Caldwell, C.C., Petzinger, G.M., Jakowec, M.W. \& Cadenas, E. Treadmill exercise rescues mitochondrial function and motor behavior in the CAG140 knock-in mouse model of Huntington's disease. Chem Biol Interact 315, 108907 (2020).

6. Stefanko, D.P., Shah, V.D., Yamasaki, W.K., Petzinger, G.M. \& Jakowec, M.W. Treadmill exercise delays the onset of non-motor behaviors and striatal pathology in the CAG140 knock-in mouse model of Huntington's disease. Neurobiol Dis 105, 15-32 (2017).

7. van Dellen, A., Cordery, P.M., Spires, T.L., Blakemore, C. \& Hannan, A.J. Wheel running from a juvenile age delays onset of specific motor deficits but does not alter protein aggregate density in a mouse model of Huntington's disease. BMC Neurosci 9, 34 (2008).

8. Harrison, D.J., et al. Exercise attenuates neuropathology and has greater benefit on cognitive than motor deficits in the R6/1 Huntington's disease mouse model. Exp Neurol 248, 457-469 (2013).

9. Trembath, M.K., et al. A retrospective study of the impact of lifestyle on age at onset of Huntington disease. Mov Disord 25, 1444-1450 (2010).

10. Fritz, N.E., et al. Physical Therapy and Exercise Interventions in Huntington's Disease: A Mixed Methods Systematic Review. J Huntingtons Dis 6, 217-235 (2017).

11. Playle, R., Dimitropoulou, P., Kelson, M., Quinn, L. \& Busse, M. Exercise Interventions in Huntington's Disease: An Individual Patient Data Meta-Analysis. Mov Disord Clin Pract 6, 567-575 (2019). 
12. Cruickshank, T.M., et al. Effects of multidisciplinary therapy on physical function in Huntington's disease. Acta Neurol Scand 138, 500-507 (2018).

13. Cruickshank, T.M., et al. The effect of multidisciplinary rehabilitation on brain structure and cognition in Huntington's disease: an exploratory study. Brain Behav 5, e00312 (2015).

14. Hendrikse, J., Kandola, A., Coxon, J., Rogasch, N. \& Yucel, M. Combining aerobic exercise and repetitive transcranial magnetic stimulation to improve brain function in health and disease. Neurosci Biobehav Rev 83, 11-20 (2017).

15. Magee, J.C. \& Grienberger, C. Synaptic Plasticity Forms and Functions. Annu Rev Neurosci 43, 95-117 (2020).

16. Mellow, M.L., Goldsworthy, M.R., Coussens, S. \& Smith, A.E. Acute aerobic exercise and neuroplasticity of the motor cortex: A systematic review. J Sci Med Sport 23, 408-414 (2020).

17. Mooney, R.A., et al. Acute aerobic exercise modulates primary motor cortex inhibition. Exp Brain Res 234, 3669-3676 (2016).

18. Smith, A.E., Goldsworthy, M.R., Garside, T., Wood, F.M. \& Ridding, M.C. The influence of a single bout of aerobic exercise on short-interval intracortical excitability. Exp Brain Res 232, 1875-1882 (2014).

19. Singh, A.M., Duncan, R.E., Neva, J.L. \& Staines, W.R. Aerobic exercise modulates intracortical inhibition and facilitation in a nonexercised upper limb muscle. BMC Sports Sci Med Rehabil 6, 23 (2014).

20. Singh, A.M., Neva, J.L. \& Staines, W.R. Acute exercise enhances the response to paired associative stimulationinduced plasticity in the primary motor cortex. Exp Brain Res 232, 3675-3685 (2014).

21. Saucedo Marquez, C.M., Vanaudenaerde, B., Troosters, T. \& Wenderoth, N. High-intensity interval training evokes larger serum BDNF levels compared with intense continuous exercise. J Appl Physiol (1985) 119, 1363-1373 (2015).

22. Stavrinos, E.L. \& Coxon, J.P. High-intensity Interval Exercise Promotes Motor Cortex Disinhibition and Early Motor Skill Consolidation. J Cogn Neurosci 29, 593-604 (2017).

23. Mang, C.S., Snow, N.J., Campbell, K.L., Ross, C.J. \& Boyd, L.A. A single bout of high-intensity aerobic exercise facilitates response to paired associative stimulation and promotes sequence-specific implicit motor learning. $J$ Appl Physiol (1985) 117, 1325-1336 (2014).

24. Andrews, S.C., et al. Intensity Matters: High-intensity Interval Exercise Enhances Motor Cortex Plasticity More Than Moderate Exercise. Cereb Cortex 30, 101-112 (2020).

25. McColgan, P., Joubert, J., Tabrizi, S.J. \& Rees, G. The human motor cortex microcircuit: insights for neurodegenerative disease. Nat Rev Neurosci (2020).

26. Orth, M., et al. Abnormal motor cortex plasticity in premanifest and very early manifest Huntington disease. $J$ Neurol Neurosurg Psychiatry 81, 267-270 (2010).

27. Philpott, A.L., et al. Cortical inhibitory deficits in premanifest and early Huntington's disease. Behav Brain Res 296, 311-317 (2016).

28. Schippling, S., et al. Abnormal motor cortex excitability in preclinical and very early Huntington's disease. Biol Psychiatry 65, 959-965 (2009).

29. Crupi, D., et al. Cortical and brainstem LTP-like plasticity in Huntington's disease. Brain Res Bull 75, 107-114 (2008).

30. Hannan, A.J. Environmental enrichment and brain repair: harnessing the therapeutic effects of cognitive stimulation and physical activity to enhance experience-dependent plasticity. Neuropathol Appl Neurobio/ 40, 13-25 (2014).

31. Rossi, S., Hallett, M., Rossini, P.M., Pascual-Leone, A. \& Safety of, T.M.S.C.G. Safety, ethical considerations, and application guidelines for the use of transcranial magnetic stimulation in clinical practice and research. Clin Neurophysio/ 120, 2008-2039 (2009). 
32. Sports Medicine Australia. Sports Medicine Australia Pre-exercise Screening System. (Government of Australia, Canberra, ACT, 2011).

33. Penney, J.B., Jr., Vonsattel, J.P., MacDonald, M.E., Gusella, J.F. \& Myers, R.H. CAG repeat number governs the development rate of pathology in Huntington's disease. Ann Neurol 41, 689-692 (1997).

34. Huntington Study Group. Unified Huntington's Disease Rating Scale: reliability and consistency. Mov Disord 11, 136142 (1996).

35. Shoulson, I. \& Fahn, S. Huntington disease: clinical care and evaluation. Neurology 29, 1-3 (1979).

36. Oldfield, R.C. The assessment and analysis of handedness: the Edinburgh inventory. Neuropsychologia 9, 97-113 (1971).

37. Craig, C.L., et al. International physical activity questionnaire: 12-country reliability and validity. Med Sci Sports Exerc 35, 1381-1395 (2003).

38. Philpott, A.L., Fitzgerald, P.B., Cummins, T.D. \& Georgiou-Karistianis, N. Transcranial magnetic stimulation as a tool for understanding neurophysiology in Huntington's disease: a review. Neurosci Biobehav Rev 37, 1420-1433 (2013).

39. Ziemann, U., et al. TMS and drugs revisited 2014. Clin Neurophysio/ 126, 1847-1868 (2015).

40. Carcea, I. \& Froemke, R.C. Cortical plasticity, excitatory-inhibitory balance, and sensory perception. Prog Brain Res 207, 65-90 (2013).

41. Huang, Y.Z., Edwards, M.J., Rounis, E., Bhatia, K.P. \& Rothwell, J.C. Theta burst stimulation of the human motor cortex. Neuron 45, 201-206 (2005).

42. Borg, G. Perceived exertion as an indicator of somatic stress. Scandinavian Journal of Rehabilitation Medicine 2, 92-98 (1970).

43. Coxon, J.P., Stinear, C.M. \& Byblow, W.D. Intracortical inhibition during volitional inhibition of prepared action. $J$ Neurophysio/ 95, 3371-3383 (2006).

44. Wagenmakers, E.J., et al. Bayesian inference for psychology. Part I: Theoretical advantages and practical ramifications. Psychon B Rev 25, 35-57 (2018).

45. Dienes, Z. Using Bayes to get the most out of non-significant results. Front Psycho/ 5(2014).

46. JASP Team. JASP. (2020).

47. Rouder, J.N., Morey, R., Speckman, P.L. \& Province, J.M. Default Bayes factors for ANOVA designs. Journal of Mathematical Psychology 56, 356-374 (2012).

48. Jeffreys, H. Theory of Probability, (Oxford University Press, Oxford, UK, 1961).

49. Lee, M.D. \& Wagenmakers, E.J. Bayesian Cognitive Modeling: A Practical Course, (Cambridge University Press, Cambridge, 2014).

50. Abbruzzese, G., et al. Intracortical inhibition and facilitation are abnormal in Huntington's disease: a paired magnetic stimulation study. Neurosci Lett 228, 87-90 (1997).

51. Muller-Dahlhaus, F. \& Ziemann, U. Metaplasticity in human cortex. Neuroscientist 21, 185-202 (2015).

52. Blumenstock, S. \& Dudanova, I. Cortical and Striatal Circuits in Huntington's Disease. Front Neurosci 14, $82(2020)$.

53. Mang, C.S., et al. Exploring genetic influences underlying acute aerobic exercise effects on motor learning. Sci Rep 7, 12123 (2017).

54. He, Y.Y., Zhang, X.Y., Yung, W.H., Zhu, J.N. \& Wang, J.J. Role of BDNF in central motor structures and motor diseases. Mol Neurobiol 48, 783-793 (2013).

55. McMorris, T., Turner, A., Hale, B. \& Sproule, J. Beyond the Catecholamines Hypothesis for an Acute ExerciseCognition Interaction: A Neurochemical Perspective. in Exercise-Cognition Interaction: Neuroscience Perspectives (ed. McMorris, T.) 65-103 (Academic Press, Elsevier, 2016). 
56. Gundersen, V., Storm-Mathisen, J. \& Bergersen, L.H. Neuroglial Transmission. Physiol Rev 95, 695-726 (2015).

57. Antal, A., et al. Brain-derived neurotrophic factor (BDNF) gene polymorphisms shape cortical plasticity in humans. Brain Stimul 3, 230-237 (2010).

\section{Figures}

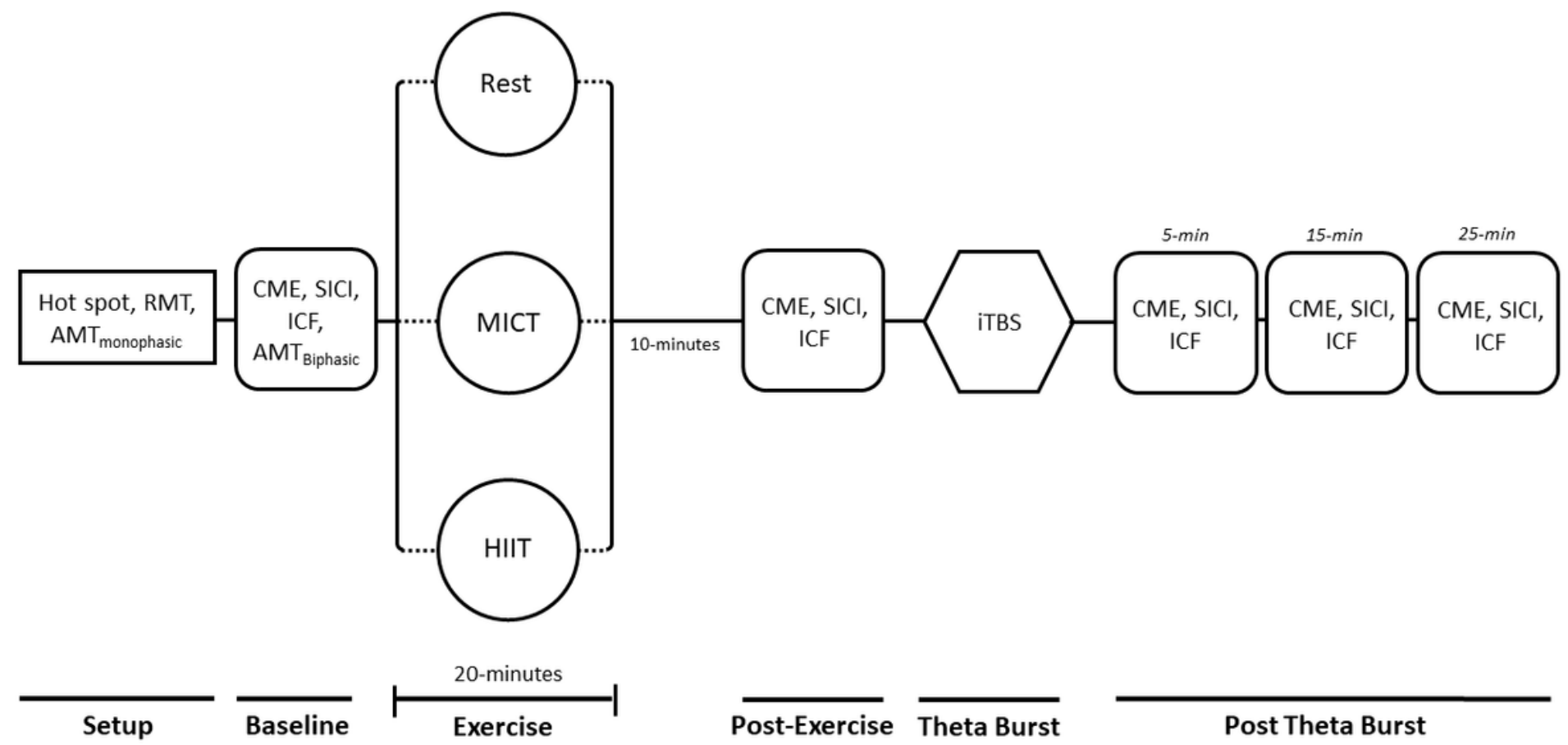

Figure 1

Sequence of events for each session. RMT - resting motor threshold; AMT - active motor threshold; CME - cortico-motor excitability; SICI - short-interval intracortical inhibition; ICF - intracortical facilitation; LICI - long-interval intracortical inhibition; HIIT - high-intensity interval training; MICT - moderate-intensity continuous training; iTBS - intermittent theta burst stimulation. Participants completed one 20-min bout of exercise, or equivalent period of rest, per session. 


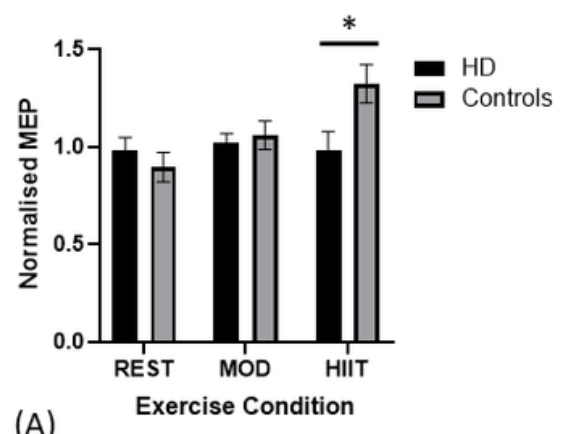

(A)

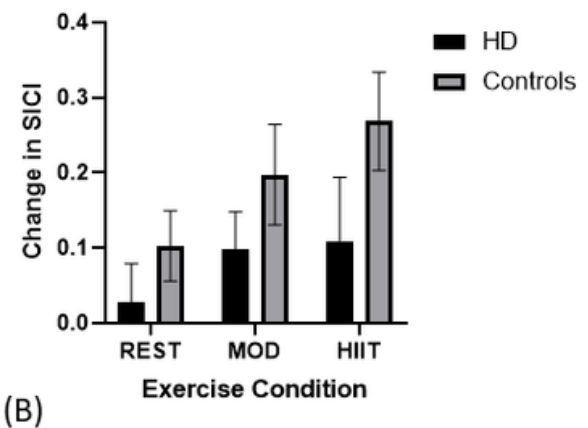

(B)

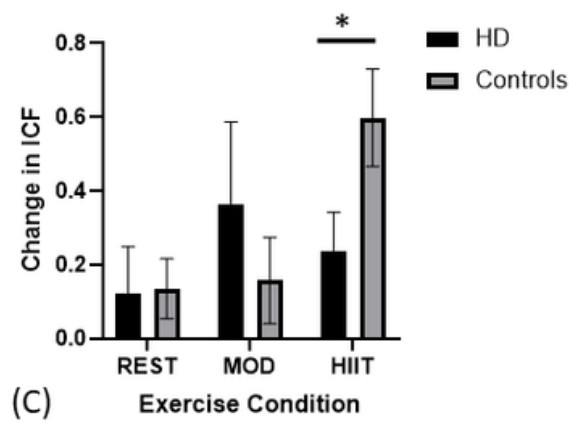

Figure 2

Effect of high-intensity interval training (HIIT), moderate-intensity continuous training (MICT) and rest on TMS measures for each group. Data represent the mean (standard error). ITBS - intermittent theta burst stimulation. Panel (A) Corticomotor excitability (CME), average post-iTBS MEP, normalised from post-exercise time-point. Panel (B) Short-interval intracortical inhibition (SICl). Change from pre-exercise baseline to average post-iTBS time-points. Panel (C) Intracortical facilitation (ICF). Change from pre-exercise baseline to average post-iTBS time-points. ${ }^{*} p<.05$. 